\title{
PONENCIA
}

\section{EVALUACIÓN DE LA CAMPAÑ̃ DE VACUNACIÓN DE LA ENFERMEDAD MENINGOCÓCICA EN GALICIA. METODOLOGÍA EMPLEADA, VENTAJAS E INCONVENIENTES. ESTUDIO DE PORTADORES}

\author{
Alberto Malvar Pintos. \\ Dirección General de Salud Pública. Consejería de Sanidad y Servicios Sociales de la Comunidad Autónoma de \\ Galicia.
}

\section{INTRODUCCIÓN}

Durante la temporada 1995/96 se observó en Galicia un importante aumento del número de casos de enfermedad meningocócica (EM), debido fundamentalmente a la nueva cepa $C: 2 b: P 1 \cdot 2,5^{1}$. La magnitud de este aumento, la distribución geográfica de los casos y la existencia de vacuna frente al serogrupo C, propició que la Dirección Xeral de Saúde Pública estudiase diferentes estrategias de control y acabase recomendando una campaña de vacunación, con carácter excepcional y dirigida a los residentes en Galicia que durante su desarrollo (diciembre de 1996 y enero de 1997) tuviesen entre 18 meses y 19 años de edad ${ }^{2}$. Así mismo, se consideró oportuno aprovechar la afluencia de los niños a los centros de vacunación para realizar un estudio de portadores de la cepa $C: 2 b: P 1.2,5$ en dos áreas, una de baja y otra de alta incidencia de EM.

En esta intervención se comenta, primero, la evolución de la EM en el primer año de seguimiento tras la campaña, 1997. Luego, lo que llamamos resultado de la campa-

Correspondencia:

D. Alberto Malvar Pintos

Dirección General de Salud Pública

Consejería de Sanidad y Servicios Sociales

Avda. Camino Francés, 10 Bajo

15771 Santiago de Compostela

Correo Electrónico: dxsp18@jet.es ña, con el que intentamos aproximar el beneficio sanitario debido a ella. Para finalizar, se recogen las conclusiones del estudio de portadores realizado a la par que la campaña de vacunación.

\section{Evolución de la enfermedad meningocócica tras la campaña de vacunación}

Según los datos disponibles en enero de 1998, durante 1997 se observaron en Galicia 10,8 casos de EM por cien mil habitantes $\left(\mathrm{c} / 10^{5} \mathrm{~h}\right)$, incidencia menor que la observada durante $1996\left(14,8 \mathrm{c} / 10^{5} \mathrm{~h}\right)$, si bien se mantuvo por encima de la de 1995 y los años anteriores (menos de $\left.5 \mathrm{c} / 10^{5} \mathrm{~h}\right)^{2}$. El descenso ocurrió fundamentalmente en la segunda mitad del año y se puede atribuir, como vemos en la tabla 1, a la disminución de la EM debida al serogrupo $\mathrm{C}$, ya que la incidencia de la debida al serogrupo B y la de los casos en los que hay sospecha clínica y no aislamiento del meningococo (en la tabla, serogrupo «desconocido»), se mantuvieron en niveles semejantes. También disminuyó la mortalidad; de 19 casos en 1996 (15 por serogrupo C) se pasó a 9 (6 de ellos por serogrupo $\mathrm{C}$ ), por lo que también en este caso parece posible atribuir su descenso al del serogrupo C. La letalidad, en cambio, no mostró un descenso tan acusado. 
La sola observación de comportamientos como el descrito nada dicen, sin embargo, de la posible responsabilidad que en ellos pudiera tener la campaña de vacunación. Descensos semejantes, de un año para otro, seguidos de un nuevo aumento de la incidencia, pudieron observarse durante la epidemia de los años 70-80². (Del mismo modo que la incidencia no hubiese variado, $o$ incluso hubiese aumentado ligeramente, no sería indicio suficiente para calificar negativamente el resultado de la campaña de vacunación).

De hecho, sólo podemos valorar el papel de la campaña, sin tener en cuenta la condición de vacunados o no vacunados de los casos y la población, mediante el estudio de la distribución por edad de los casos de EM, ya que es ésta la única característica relacionada con la campaña. (Consideramos que, por su baja frecuencia, carecen de relevancia poblacional las inmunopatías en las que está recomendada la vacunación ${ }^{3}$ ).

La distribución por edad de los casos de EM es muy característica ${ }^{4}$. Las mayores incidencias ocurren en los menores de cinco años, especialmente en los más pequeños, y suelen suponer alrededor de la mitad de los casos. Por otro lado, cerca de $2 / 3$ de éstos suelen ocurrir en el grupo de edad que llamaremos «vacunable» (2 a 19 años). Como vemos en la tabla 1, éstas son también las características de la distribución por edad observada en Galicia durante 1996, tanto para toda la EM como para la debida al serogrupo C. Asimismo, distribuciones semejantes fueron observadas en otras comunidades en las que la incidencia de EM también aumentó debido a la C:2b:P1.2,5

La distribución por edad de los casos de EM observada en 1997, también expuesta en la tabla 1, es sólo ligeramente diferente a la observada en 1996, si bien con incidencias menores en todos los grupos de edad, salvo en los mayores de 19 años, que duplican su incidencia y triplican su participación en la enfermedad. La distribución por edad de los casos de enfermedad debida al serogrupo $\mathrm{C}$ es, en cambio, radicalmente diferente: más del $40 \%$ de los casos ocurren en menores de 2 años, un $25 \%$ en mayores de 19 y en edades vacunables sólo 1/3 de ellos, mientras en 1996 habían alcanzado los $2 / 3$. También en Cantabria ${ }^{5}$ y La Rioja ${ }^{7}$, tras la campaña de vacunación, se observó la misma alteración en la distribución por edad de los casos debidos al serogrupo $\mathrm{C}$.

La importante discrepancia entre las distribuciones de edad de la EM considerada en

Tabla 1

Enfermedad meningocócica en Galicia. Años 1996 y 1997

\begin{tabular}{|c|c|c|c|c|c|c|c|c|c|c|c|c|}
\hline \multirow{4}{*}{$\begin{array}{l}\text { Srgr. Let." } \\
\text { Edad (años) }\end{array}$} & \multicolumn{6}{|c|}{ Año 1996} & \multicolumn{6}{|c|}{ Año 1997} \\
\hline & \multicolumn{6}{|c|}{$\begin{array}{l}\mathrm{B}=1.3 ; \mathrm{C}=6.3 ; \text { desconocido }=6.8 \\
\mathrm{Lg}=4,8 \% ; \mathrm{Lc}=8.7 \%\end{array}$} & \multicolumn{6}{|c|}{$\begin{array}{l}\mathrm{B}=1,4: \mathrm{C}=3,3: \text { desconocido }=6,0 \\
\mathrm{Lg}=3,0 \%: \mathrm{Lc}=6.8 \%\end{array}$} \\
\hline & \multicolumn{3}{|c|}{ Toda la EM } & \multicolumn{3}{|c|}{ Serograpo $C$} & \multicolumn{3}{|c|}{ Toda la EM } & \multicolumn{3}{|c|}{ Serogrupo $C$} \\
\hline & $\cos c o s$ & tasis $a^{\circ}$ & $\%$ & $\cos \theta s$ & $t a s t^{2}$ & $\%$ & casos & tascis & $\%$ & cassos & $\operatorname{tasat}^{\mathrm{c}}$ & $\%$ \\
\hline$<2$ & 88 & 222 & 22 & 44 & $1 \mathrm{il}$ & 26 & 74 & 188 & 25 & 37 & 94 & 42 \\
\hline $2-4$ & 107 & 166 & 27 & 42 & 65 & 24 & 63 & 98 & 22 & 13 & 20 & 15 \\
\hline $5-9$ & 84 & 71 & 21 & 28 & 24 & 16 & 64 & 54 & 22 & 6 & 5 & 7 \\
\hline $10-14$ & 47 & 30 & 12 & 18 & 11 & 10 & 18 & 11 & 6 & 2 & 1 & 2 \\
\hline $15-19$ & 42 & 20 & 11 & 25 & 12 & 15 & 21 & 10 & 7 & 9 & 4 & 10 \\
\hline$>19$ & 26 & 1 & 7 & 15 & 1 & 9 & 52 & 2 & 18 & 21 & 1 & 24 \\
\hline Total & 394 & 14.8 & 100 & 172 & 6.3 & 100 & 292 & 10,8 & 100 & 88 & 3.3 & 100 \\
\hline
\end{tabular}

(a) Serogrupos: tasas hrutas por $105 \mathrm{~h}$ : (b) Letalitad: general - l.g- y por serogrupo $\mathrm{C}$. $\mathrm{Cl}$ : (c) $\mathrm{d} / 10^{5} \mathrm{~h}$. 
conjunto y de la debida a serogrupo $\mathrm{C}$, podría deberse a un fenómeno que dimos en llamar «subdetección de meningococos en vacunados» y que caracterizamos con las cuatro observaciones siguientes: (i) en los no vacunables (ie, los que no tenían -durante la campaña, la edad en la que se recomendaba la vacunación), la proporción de casos en los que la sospecha de EM se confirmó microbiológicamente (capacidad de «detección») fue, en 1997, la misma que en 1996; (ii) en los no vacunados, la capacidad de detección observada en 1997 también fue semejante a la observada en 1996 en ese mismo intervalo de edad; (iii) en los vacunados, la capacidad de detectar serogupo B en 1997 fue consistente con la observada en 1996 en su mismo intervalo de edad; (iiii) en los vacunados, excluidos los casos debidos al serogrupo B, la capacidad de detección observada en 1997 fue cuatro veces menor que la observada en 1996 en su mismo intervalo de edad. Esta concentración de la subdetección en vacunados, unida a que la cantidad y la distribución geográfica de los casos no parecían fácilmente explicables por otro microorganismo, nos hizo considerar la posibilidad de que la vacuna, además de evitar cualquier manifestación de enfermedad en algunos individuos, pudiera en otros entorpecer la evolución clínica dificultando, además, el aislamiento del meningococo. Por este motivo, se ha iniciado un estudio con la intención de determinar el carácter meningocócico de esos casos (mediante PCR) y, si éste se confirma, comparar la gravedad clínica y la capacidad bactericida del suero de vacunados y no vacunados.

\section{EVALUACIÓN DEL RESULTADO DE LA CAMPAÑA}

Evaluación directa y poblacional. Consideraciones como la precedente sólo nos permiten afirmar, y ello asumiendo unas determinadas condiciones de variación en la distribución por edad de la EM, que la campaña de vacunación ha sido efectiva. Sin embargo, el objetivo de la campaña es evitar que se produzcan casos de enfermedad (o muertes, o que se ganen APVP, o, ahora lo vemos, si se constata el carácter meningocócico de la subdetección, disminuir la gravedad de los casos); por lo que el resultado de la misma ha de ser evaluado en estos términos. Otras medidas relacionadas con la campaña, como la efectividad de la vacunación (que habla de la eficacia de la vacuna y las condiciones en que fue aplicada) o la cobertura (que evalúa, por ejemplo, los medios empleados en la captación de vacunados), son también interesantes, pero por sí mismas no son el resultado de la campaña. Es más, nosotros las consideramos como componentes de este resultado, al que, entre otras conceptualizaciones posibles, entendemos constituido por tres elementos: la cobertura vacunal, la efectividad de la vacuna y la incidencia de la enfermedad observada durante el seguimiento.

Si la vacuna sólo afecta a la susceptibilidad del vacunado y no interfiere la transmisión de la infección, estos tres elementos contribuyen al resultado en cl mismo sentido, de forma proporcional y de modo independiente. De tal suerte que el resultado será mejor, se evitarán más casos, cuanto mayor sea la cobertura (hay mayor número de individuos protegidos), la efectividad de la vacuna (es major la protección) y la incidencia de la enfermedad (hay más casos que evitar). Este modo de entender el resultado de una campaña de vacunación, que fue el que se utilizó en su momento para decidir entre diferentes estrategias de control, conduce a que la evaluación se realice desde una perspectiva que llamaremos directa, basada en la comparación de la incidencia de enfermedad en los vacunados y los no vacunados. (Es el diseño tipo I de Halloran y Struchiner ${ }^{8}$ ).

En cambio, si la vacuna además de afectar la susceptibilidad interfiere en la transmisión de la infección, los tres elementos mencionados pierden su independencia, ya que la vacunación de unos disminuiría las posibilidades de enfermar de quienes no se vacunan, debido a que será menor la probabilidad de 
mantener un contacto efectivo desde el punto de vista de la transmisión ${ }^{9}$. En estos casos, por efecto de la inmunidad de grupo, mayores coberturas producirán incidencias menores en los no vacunados, por lo que no se podrá evaluar el resultado de una campaña sólo con la información obtenida en los miembros de la población en la que se llevó a cabo -que lo subestimaría; habrá que adoptar, pues, una perspectiva diferente, que llamaremos poblacional, en la que es necesaria información de lo ocurrido en una población diferente. (Es el diseño III de Halloran y Struchiner ${ }^{8}$ ).

Esta última perspectiva de evaluación es la que, creemos, conviene a la enfermedad meningocócica, ya que se ha observado que la vacuna frente al serogrupo $C$ reduce la duración del estado de portador ${ }^{10}$, lo que bien podría disminuir la probabilidad de exposición efectiva de los contactos no vacunados. Sin embargo, carecemos de una población control que sea comparable con la población en la que se realizó la campaña; es decir, que sean semejantes tanto en las caractcrísticas que determinan la difusión de la infección (de tal modo que la población control esté sometida, durante todo el período de seguimiento, al riesgo de enfermedad que se habría observado en la población en la que se realiza la campaña si ésta no se hubiese llevado a cabo), como en la distribución de los factores que determinan la susceptibilidad a la enfermedad. Las poblaciones, además, no deben interactuar en términos de transmisión, y en ellas la definición y búsqueda de casos han de ser similares $^{11}$.

En nuestro caso, lamentablemente, no podemos utilizar como control ninguna población diferente a la de Galicia (las de nuestro entorno tradicionalmente han manifestado intensidades diferentes de EM y, en la actualidad, están vacunadas en mayor o menor medida), ni podemos utilizar tampoco sus antecedentes históricos, ya que la cepa es necesariamente diferente a las del serogrupo $\mathrm{B}$ que eran responsables de la EM en años anteriores, las condiciones sociales que permiten la difusión de la infección cambiaron y, además, la metodología de predicción son ineficientes a un plazo tan largo como el que parece exigir la enfermedad. Para finalizar, se ha propuesto un método para estimar la incidencia de la población control a partir de la incidencia observada en la población de la campaña, pero la EM no cumple al menos dos de los supuestos del método ${ }^{12}$ : que infección y enfermedad coincidan, y que siga un modelo de transmisión del tipo SusceptibleInfectado-Recobrado, puesto que se puede adquirir la condición de resistente sin haber sido infectado, debido a la inmunización cruzada por $N$. lactamica o por cepas de meningococos no-capsulados ${ }^{10}$.

Por lo tanto, al no poder realizar una evaluación poblacional del resultado de la campaña, tendremos que conformarnos con una evaluación directa, y tener presente que da lugar a una subestimación del resultado poblacional en presencia de inmunidad de grupo.

Evaluación directa del resultado. La evaluación directa del resultado se basa en la comparación de la incidencia de la enfermedad en vacunados y no vacunados, miembros ambos de una misma población. Para evitar que la evaluación resulte sesgada, habrá que asumir, además de que la población es cerrada a efectos de la transmisión, que la exposición de vacunados y no vacunados es comparable, que la vacunación es «aleatoria» y que el efecto de la vacuna es homogéneo ${ }^{13}$.

El diseño que mejor garantizaría la comparabilidad de la exposición entre vacunados y no vacunados, sería alguno de los basados en la medición de las tasas de ataque secundarias en grupos de mezcla de tamaño pequeño (hogares, aulas, colegios, etc... $)^{14}$; pero, en la EM, los casos secundarios ocurren con una frecuencia muy baja ${ }^{15} \mathrm{y}$, además, las estrategias de control de la enfermedad van dirigidas precisamente a evitar que ocurran $^{3}$. No pudiendo controlar la exposi- 
ción con el discño, la cvaluación ha de afrontar dos problemas, la baja incidencia de la enfermedad y la posible existencia de bolsas de no vacunados.

Se ha afirmado, al parecer de modo arbitrario, que es necesaria una tasa de ataque de al menos un $5 \%$ durante el seguimiento, para garantizar la comparabilidad en la exposición de vacunados y no vacunados ${ }^{16}$. En simulaciones que utilizan el SIR como modelo de transmisión, se ha observado que ese umbral, en general insuficiente, varía según ciertas características de la población, y que la exigencia es menor a medida que aumenta la inmunidad de fondo, bien de forma natural o por vacunación ${ }^{16}$. Estas consideraciones son comprometedoras cuando se trata de EM, ya que su incidencia es netamente inferior a la propuesta, aun considcrando los grupos de edad con mayor riesgo. Sin embargo, aunque pueden no ser del todo generalizables los hallazgos obtenidos con un modelo SIR, que la EM no cumple, la cobertura en Galicia es elevadísima, especialmente en quienes, entre los vacunables, tienen mayor riesgo de enfermar, por lo que no nos parece que la baja incidencia de la EM suponga un problema grave para la comparabilidad en la exposición de vacunados y no vacunados ${ }^{3}$.

Este mismo argumento, la elevada cobertura alcanzada, sirve para relativizar la influencia de las posibles bolsas de no vacunados que pudiesen existir, y que tendrían mayor o menor relevancia dependiendo de la inmunidad de grupo que pueda generar la vacuna. Pero, además, el problema que podrían suponer las bolsas de vacunados se ve disminuido también porque el protocolo de control de la EM recomienda la vacunación de los no vacunados del entorno de los casos.

Por vacunación aleatoria se entiende que la decisión de vacunarse ha de ser independiente de la susceptibilidad individual, de tal modo que los grupos vacunado y no vacunado sean igualmente suscepti- bles. Como en la EM, salvo la edad, sólo ciertas inmunopatías indican una susceptibilidad diferente, y estas son raras y en todo caso indican la vacunación, podemos asumir que no habrá diferencias de susceptibilidad en favor de los vacunados. La edad, se trata en el análisis.

Un problema difícil de catalogar como relacionado con la exposición o la susceptibilidad, es el definido por la variación de la incidencia de enfermedad en las diferentes estaciones del año y también en el espacio. La catalogación es difícil por la falta de relación estacional entre la prevalencia de portadores y la incidencia de la enfermedad ${ }^{10}$, así como por la ausencia de un conocimiento preciso del por qué enferman los que enferman y otros no lo hacen ${ }^{10}$. En Galicia, como la campaña duró cerca de dos meses y se realizó en la época de mayor ricsgo, bastantes individuos vivieron como no vacunados el momento de más riesgo para, luego, una vez vacunados, experimentar como tales la época del año con menor riesgo de enfermedad. Para tratar este problema utilizamos un denominador en personas-semana. Es menor el problema que plantean las diferencias geográficas del riesgo, ya que la cobertura vacunal es uniforme en todo el territorio gallego.

Para finalizar, las vacunas utilizadas no tienen efecto homogéneo: su capacidad de protección y la duración de ésta varían con la edad ${ }^{17}$. El efecto de estas diferencias intentaremos controlarlo especificando el resultado observado en diferentes grupos de edad, y analizándolo en períodos de un año de duración. La presente exposición hace referencia al primero de estos períodos.

Métrica. Entendido como casos evitados, el resultado de la campaña lo definimos como el número de casos que habrían de ocurrir en los vacunados para que la incidencia de la EM en ellos fuese igual a la observada entre los no vacunados. Para su cálculo habremos de disponer de datos sobre la inci- 
dencia de la enfermedad, sobre el estado vacunal de los casos y sobre la distribución de la vacunación en la población.

La incidencia de la enfermedad se conoce por la vigilancia activa diaria que, sin interrupción, se desarrolla en los hospitales de Galicia desde el comienzo de la temporada 95/96. La vigilancia se basa en la sospecha clínica (meningitis bacteriana, petequias), tras la que se estudia el estado vacunal del caso. En principio, la sospecha debería ser independiente de la condición de vacunado o no vacunado del enfermo, ya que surge tras conocer los resultados del LCR y/o la presencia de petequias. (La independencia entre sospecha y estado vacunal, que se exige para que no se dé un sesgo de sobrevaloración de la vacuna, quizá se vea afianzada en nuestro caso puesto que, como veremos, la efectividad de la vacuna es mayor si se mide sólo con los casos confirmados, circunstancia semejante a la ocurrida recientemente en Canadá en el primer año de seguimiento ${ }^{18}$ ). Se utilizan dos definiciones de caso, una «específica» y otra "sensible» que se exponen en la tabla 2, al igual que las de caso vacunado y caso no vacunado.

La distribución de la vacunación en la población la conocemos por el sistema de registro numérico empleado en la campaña. Según él, todos los puntos de vacunación remitirían, cada semana, el número de individuos vacunados en los siguientes grupos de edad: menores de 3 años (donde se acabó alcanzando una cobertura del $86 \%$ ), de 3 a 5 años (cobertura final del $99 \%)$, de 6 a 13 años (97\%), de 14 a 17 años $(84 \%)$ y de 18 y 19 años $(47 \%)$. La cobertura total al final de la campaña fue del $86 \%$. En este apartado, carecemos de información sobre los que, en edad vacunable y no vacunados en su mayoría, viajaron a Galicia durante 1997. Para incluirlos en el análisis habríamos de disponer de su número, distribución por edad y momento y duración de la estancia, para traducirlo en personas semanas. Sin embargo, como su magnitud es relativamente pequeña y en su mayoría acuden en la época en la que se ha observado un riesgo menor de enfermedad, consideramos que su exclusión en nada altera el análisis.

En la tabla 2 se recogen, divididas en tres grupos de edad (los anteriores, tras excluir los menores de 2 años, porque se hará un análisis separado, y agrupando los de 14 a 19 años, puesto que en un grupo no hay casos del serogrupo C) y las poblaciones de personas-semana vacunadas y no vacunadas acumuladas en el transcurso de 1997. También se recogen, para las definiciones específica y sensible, el número de casos y la incidencia por cien mil en vacunados y no vacunados, así como la efectividad vacunal y los casos evitados.

Tabla 2

Resultado (provisional) de la campaña tras un año de seguimiento

\begin{tabular}{|c|c|c|c|c|c|c|c|c|c|c|c|c|c|c|}
\hline \multirow{3}{*}{ Edad (años) } & & & \multicolumn{6}{|c|}{ Definición especifica ${ }^{a}$} & \multicolumn{6}{|c|}{ Definición sensible ${ }^{\mathrm{b}}$} \\
\hline & \multicolumn{2}{|c|}{ Población $^{\mathrm{c}}$} & \multicolumn{2}{|c|}{ casos } & \multicolumn{2}{|c|}{ tasa } & \multirow[b]{2}{*}{$E V^{t}$} & \multicolumn{3}{|c|}{ casos } & \multicolumn{2}{|c|}{ tasa } & \multirow[b]{2}{*}{$E V$} & \multirow[b]{2}{*}{$C E$} \\
\hline & vac & no & $v a c^{d}$ & $n o^{\mathrm{d}}$ & vac & no & & $C E^{\mathrm{g}}$ & vac & no & $v e c$ & no & & \\
\hline $2-5$ & 4276687 & 251632 & 6 & 10 & 5.1 & 143 & 96 & 164 & 39 & 16 & 33 & 229 & 86 & 233 \\
\hline $6-3$ & 10857802 & 248136 & 1 & 4 & 0,3 & 58 & 99 & 174 & 62 & 6 & 21 & 87 & 76 & 201 \\
\hline $14-19$ & 8856297 & 4155309 & 1 & 8 & 0.4 & 7 & 94 & 16 & 6 & 13 & 3 & 11 & 78 & 22 \\
\hline SUMA & & & 8 & 22 & & & & 354 & 107 & 35 & & & & \\
\hline
\end{tabular}

(a) Definición específica: caso del serogrupo $\mathrm{C}$; ie, aislamiento de meningococo en lugar normalmente estéril:

(b) Definición sensible: caso del serogrupo Co sospecha clínica de EM; (c) pohlación en personas-semana:

(d) Caso vacunado: si recibió la vacuna y enfermó después de 15 días; (c) caso no vacunado: siendo vacunable, no recibió la vacuna o lo hizo y enfermó antes de 5 días; (f) FV: efectividad vacunal, expresada en porcentaje ; $(g)$ CE: casos evitados. 


\section{COMENTARIO}

Haber medido el resultado de la campaña como casos evitados permite estimar la magnitud del beneficio alcanzado tras la campaña de vacunación, además de que hace posibles ulteriores estudios de carácter económico. Sin embargo, el valor concreto del resultado hay que matizarlo. Primero, en su definición no hay correspondencia con lo que habría ocurrido durante 1997 si no se hubiese realizado la vacunación, auténtico referente de la evaluación de la campaña, especialmente si la vacuna produce inmunidad de grupo. Además, su valor depende en gran medida de la estratificación utilizada. La que presentamos fue impuesta por el sistema de información de la campaña, que iba dirigido a conocer sus progresos semana a semana, para poder intervenir si fuese necesario. De todos modos se corresponde bastante bien con lo que sabemos sobre la distribución de la susceptibilidad a la EM y del comportamiento de la vacuna.

El valor del resultado podría, también, infra o sobrevalorar el papel de la campaña, si hubiese errores en la medición de la incidencia o el estado vacunal de vacunados y no vacunados, así como si estos grupos no son comparables en exposición o susceptibilidad. Por último, recordamos que esta cvaluación cs sólo la primera de una serie con la que se podrá tener mejor información sobre el comportamiento de la vacuna (que pierde eficacia con el paso del tiempo) y el resultado de la campaña.

Teniendo presente lo dicho, creemos que se puede concluir que durante el primer año la campaña ha obtenido un buen resultado, incluso superior al establecido como objeti$v^{19}{ }^{19}$, aun considerando la definición específica. En cuanto a la efectividad, también se mantuvo en los niveles esperados, si bien más bajos cuando utilizamos la definición sensible. La discrepancia cntre la efectividad, que es mayor cuando utilizamos la definición específica, y el resultado, que es mayor cuando usamos la definición sensible, se debe a la mayor cantidad de enfermedad que asume la definición sensible, y a que ese exceso de enfermedad, que en parte fue descrito como subdetección, ocurre preferentemente en vacunados.

\section{Estudio de Portadores}

Como se dijo, durante la campaña de vacunación se realizó, también, un estudio de portadores de la cepa epidémica. Se tomaron dos muestras independientes entre los que acudían a vacunarse, una en la zona de alta incidencia y otra en la de baja. El estudio microbiológico fue diseñado y realizado por el Laboratorio de Meningococos del Centro Nacional de Microbiología.

Como el diseño del estudio ya fue resumido en otro lugar ${ }^{20}$, se resumen ahora sus conclusiones, apoyándolas con la tabla 3 , donde sc exponen las prevalencias observadas en los distintos grupos de edad en las dos áreas:

\section{Conclusiones del estudio de portadores}

- La prevalencia de portadores de la cepa C: $2 \mathrm{~b}: \mathrm{P} 1.2,5$ es muy baja, $0,6 \%$ ( $\mathrm{IC}_{95 \%}$ : $0,28 \%-0,93 \%$ ) en la zona de alta incidencia y $0,41 \%\left(\mathrm{IC}_{95 \%}: 0,00 \%-1,04 \%\right)$ en la de baja.

- No se pudo descartar la igualdad de la prevalencia de portadores en ambas áreas, de baja y alta incidencia de enfermedad, pero puede ser efecto del diseño utilizado ${ }^{19}$.

- No se encontraron portadores de cepa epidémica en los de 2 a 4 años de edad.

- La prevalencia del serogrupo B es significativamente mayor que la del C.

- La distribución por edad de las $N$. lactamica y N. meningitidis es la habitual. 
Tabła 3

\begin{tabular}{|c|c|c|c|c|c|c|}
\hline & Are & & $\begin{array}{c}N \text { meningitidis } \\
\text { serogrupo } B\end{array}$ & $\begin{array}{l}N \text { meningitidis } \\
\text { serogrupo } C\end{array}$ & $\begin{array}{c}\text { cepa } \\
C: 2 b: P 1.2,5\end{array}$ & N lactamica \\
\hline \multirow{4}{*}{$2-4$} & Alta & $\%$ & 1.54 & 0.22 & 0.00 & 17.04 \\
\hline & & IC $95 \%$ & $0.43-2.64$ & $0.00-0.68$ & - & $11.05-23.03$ \\
\hline & Baja & $\%$ & 1.34 & 0.00 & 0.00 & 16.75 \\
\hline & & IC $95 \%$ & 0.342 .33 & - & - & $10.88-22.63$ \\
\hline \multirow{4}{*}{$5-9$} & Alta & $\%$ & 2.34 & 0.45 & 0.19 & 7.22 \\
\hline & & IC $95 \%$ & $1.13-3.54$ & $0.02-0.88$ & $0.00-0.49$ & $4.12-10.33$ \\
\hline & Baja & $\%$ & 4.09 & 0.18 & 0.00 & 9.56 \\
\hline & & IC $95 \%$ & $2.81-5.38$ & $0.00-0.46$ & -- & $6.92-12.21$ \\
\hline \multirow{4}{*}{$10-14$} & Alta & $\%$ & 5.42 & 1.61 & 0.62 & 3.76 \\
\hline & & IC 95\% & $2.47-8.37$ & $0.08-3.13$ & $0.00-1.44$ & $2.36-5.17$ \\
\hline & Baja & $\%$ & 3.89 & 0.50 & 0.33 & 4.52 \\
\hline & & IC $95 \%$ & $2.61-5.16$ & $0.00-1.10$ & $0.00-0.91$ & $2.82-6.23$ \\
\hline \multirow{4}{*}{$15-19$} & Alta & $\%$ & 9.04 & 1.77 & 1.06 & 1.72 \\
\hline & & IC $95 \%$ & $6.49-11.59$ & $0.92-2.61$ & $0.41-1.64$ & $0.76-2.68$ \\
\hline & Baja & $\%$ & 8.09 & 1.84 & 0.79 & 2.75 \\
\hline & & IC $95 \%$ & $6.34-9.84$ & $0.11-3.58$ & $0.00-1.98$ & $0.00-5.79$ \\
\hline
\end{tabular}

\section{BIBLIOGRAFÍA}

1. A enfermidade meningocócica en Galicia: tempada 1995/96. Bol Epidemiol Galicia 1996; IX, (4).

2. A campaña de vacinación fronte o meningococo C: resultados e primeira avaliación. Bol Epidemiol Galicia 1996; IX, (6).

3. Protocolo de actuación fronte á enfermidade meningocócica invasiva, adaptado ó período que segue á vacinación masiva. Guías de Saúde Públic; 1997. Serie I. Informe I. Santiago de Compostela: Dirección Xeral de Saúde Pública. Consellería de Sanidade e Servicios Sociais; 1997.

4. Jones D. Epidemiology of Meningococcal Disease in Europe and USA. En Kartwright K, de. Meningococcal Disease. Wiley; 1995. p. 147-157.

5. Consejería de Sanidad y Servicios Sociales. Comunidad de Madrid. Informe: La enfermedad meningocócica en la comunidad de Madrid, 1997. Bol Epidemol Comunidad de Madrid 1997; 5, (4).

6. Gobierno de la Rioja. Campaña de vacunación frente al meningococo $\mathrm{C}$ en La Rioja y evaluación del efecto a 30 de septiembre de 1997. Bol Epidemiol 1997.

7. Halloran ME, Struchiner CJ. Study Designs for Dependent Hapenings. Epidemiol 1991; 2:331-8.

8. Halloran ME, Struchiner CJ, Longini MI. Study Designs for Evaluating Different Efficay and Effectiveness Aspects of Vacines. Am I Epidemiol 1997; 146:789-803.
9. Cartwright K. Meningococcal Carriege and Disease. En Kartwright K, de. Meningococcal Disease. Wiley; 1995. p. 115-46.

10. Haber M, Watelet L, Halloran ME. On Individual and Population Effectiveness. Epidemiol 1995, 24:1249-60.

11. Haber M. Estimation of the Population Effectiveness of Vaccination. Stat Med 1997; 16:601-10).

12. Chen RT, Orenstein WA. Epidemiologic Methods in Inmunization Programs. Epidemiol Rev 1996, 18: $99-117$.

13. Longini Jr MI, Halloran ME, Haber M, Chen RT. Measuring Vaccine Efficacy from Epidemics of Acute Infectious Agents. Stat Med: 1993, 12:249-63.

14. Begg N. Oulbreak Management. En Kartwrighlt K, de. Meningococcal Disease. Wiley; 1995.p. 285-305.

15. Minimun Attack rate for Measuring Measles Vaccine Efficacy. Int J Epidemiol 1995, 24:834-41.

16. Frash CE. Meningococcal Vaccines: Past, Present and Future. En Kartwright K, de. Meningococcal Disease. Wikley; 1995, p. 255-83.

17. De Wals $\mathbf{P}$ et al. Impact of mass inmunization campaign against serogroup $C$ meningococcus in the Province of Quebec, Canadá. Bull WHO 1996, 74:407-11.

18. Campaña de vacinación fronte ó meningococo do serogrupo C. Bol Epidemiol Galicia 1996. IX, núm 4.

19. Prevalencia de portadores de $\mathbf{N}$ meningitidis e incidencia de enfermedad meningocócica. Bol Fpidemiol Galicia 1997, vol X, n. ${ }^{\circ} 3$. 